\title{
Human rabies postexposure prophylaxis and rabid terrestrial animals in Ontario, Canada: 2014-2016
}

\author{
Middleton ${ }^{1 \star}$, L Friedman ${ }^{1}$, S Johnson 1 , S Buchan 1 , B Warshawsky ${ }^{1}$
}

\section{Abstract}

Background: The number of rabid terrestrial animals in Ontario has decreased markedly since the 1970s and 1980s. However, the number of recommended rabies postexposure prophylaxis (RPEP) courses has not decreased proportionally. The decision to recommend RPEP for terrestrial animal exposures should be based on a risk assessment that considers the prevalence of rabies in these animals within a jurisdiction, among other factors.

Objective: To explore trends in RPEP recommendations for exposures to terrestrial animals in Ontario in relation to the recency of terrestrial animal rabies cases by public health unit (PHU) jurisdiction.

Methods: RPEP recommendation data for the 36 Ontario PHUs were obtained from the Ontario integrated Public Health Information System and animal rabies data by PHU were obtained from the Ministry of Natural Resources and Forestry. We calculated the annual RPEP recommendation rates for terrestrial animals by PHU for 2014 to 2016, and plotted the 2016 rates in relation to the year of the most recently identified rabid terrestrial animal in the PHU.

Results: Between 2014 and 2016, the annual RPEP recommendation rates for terrestrial animal exposures by PHU ranged from 3.0 to 35.2 per 100,000 persons, with a median of 11.9 RPEP recommendations per 100,000 persons. In 2016, ten PHUs had not identified a rabid terrestrial animal in their jurisdiction for more than 15 years. Five of these PHUs had RPEP recommendation rates above the provincial median.

Conclusion: Along with other factors, consideration of the occurrence of rabies in terrestrial animals in a jurisdiction can assist in the risk assessment of dogs, cats or ferrets that are not available for subsequent observation.
This work is licensed under a Creative Commons Attribution 4.0 International License.

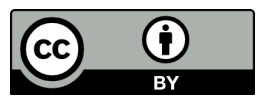

Affiliation

${ }^{1}$ Public Health Ontario, Toronto, $\mathrm{ON}$

*Correspondence:

dean.middleton@oahpp.ca

Suggested citation: Middleton D, Friedman L, Johnson S, Buchan S, Warshawsky B. Human rabies postexposure prophylaxis and rabid terrestrial animals in Ontario, Canada: 2014-2016. Can Commun Dis Rep 2019;45(7/8):177-82. https://doi.org/10.14745/ccdr.v45i78a02

Keywords: rabies postexposure prophylaxis, animal rabies, rabies risk assessment

\section{Introduction}

Rabies in humans as a result of exposure to a terrestrial animal has not been identified for over 50 years in Canada (personal communication, Stevenson B. Ontario Ministry of Natural Resources and Forestry, August 22, 2017). Nevertheless, given the almost universally fatal effect of rabies infection once symptoms develop, rabies from terrestrial animals continues to be a public health concern. In Ontario, Canada the number of terrestrial animals identified with rabies has been very low in recent years (Figure 1) with two exceptions. These exceptions were an epizootic of the raccoon strain of rabies in central west Ontario, which was identified in late 2015 and originated from a translocated raccoon, and an ongoing enzootic of the Arctic fox strain of rabies in south west and central west Ontario (1). The marked decrease in terrestrial animal rabies in Ontario has been attributed to the wildlife rabies vaccination program run by the Ontario Ministry of Natural Resources and Forestry, which began 
Figure 1: Animal rabies cases by animal type: Ontario, 2006-2017

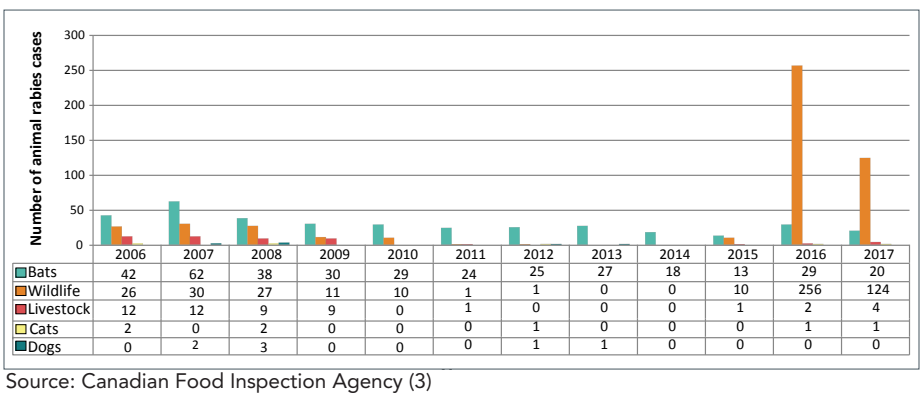

in 1989. As an additional measure to prevent human rabies cases in Ontario, rabies postexposure prophylaxis (RPEP) is publicly funded and readily available (2).

Despite the rarity of human rabies cases in Ontario, and the marked decrease in numbers of rabid terrestrial animals, the annual number of recommended RPEP courses has not decreased proportionally (4-6) (Figure 2). Administering RPEP when not indicated incurs the risk of adverse events as well as costs without benefit. One study, in the context of very low incidence of animal rabies cases, calculated that the risk of mortality from an automobile accident en route to receiving RPEP was greater than the risk of mortality from rabies (7).

According to the Ontario Rabies Prevention and Control Protocol, the decision to recommend RPEP for terrestrial animal exposures should be based on a risk assessment that considers a number of factors including the prevalence of rabies in these animals within a jurisdiction $(8,9)$. Staff at the 35 public health units (PHUs) in Ontario (36 before May 1, 2018) conduct a risk assessment after a person is exposed to an animal that could carry rabies, although ultimately the health care provider decides whether to recommend RPEP. When a dog, cat or ferret bites or scratches a person, the animal is placed under observation for 10 days by the PHU. If the animal is healthy at the end of the 10-day postexposure period, it is considered not capable of having transmitting rabies at the time of the exposure. In such cases, RPEP is not indicated.

Prior to 2013, Canadian and Ontario guidelines recommended RPEP in all instances when the dog, cat or ferret was not available for a 10-day observation period. In 2013, the guidelines were updated to recommend conducting a risk assessment to determine if RPEP is indicated after exposure to the animal. Elements of the risk assessment for a terrestrial animal that is not available for observation include the type of exposure (i.e. bite, non-bite), the anatomical location of the exposure, the risk of rabies in the animal species involved, the presence of rabies in the area where the incident occurred, and the exposure circumstances (i.e. provoked or unprovoked exposures), as well as the reliability of the injured person's history $(8,10,11)$.

In this article, we explore how local terrestrial animal rabies occurrences relate to the rate of RPEP recommendations by each PHU. For this analysis, we compare 2016 RPEP recommendation rates for terrestrial animal exposures for each PHU to the year in which the last rabid terrestrial animal was identified in each jurisdiction.

Figure 2: Annual number of animal rabies cases (terrestrial animals and bats) ${ }^{a}$ and the annual number of rabies postexposure prophylaxis courses administered and/or recommended (including exposures outside of Ontario) ${ }^{b}$, Ontario, 1958-2017
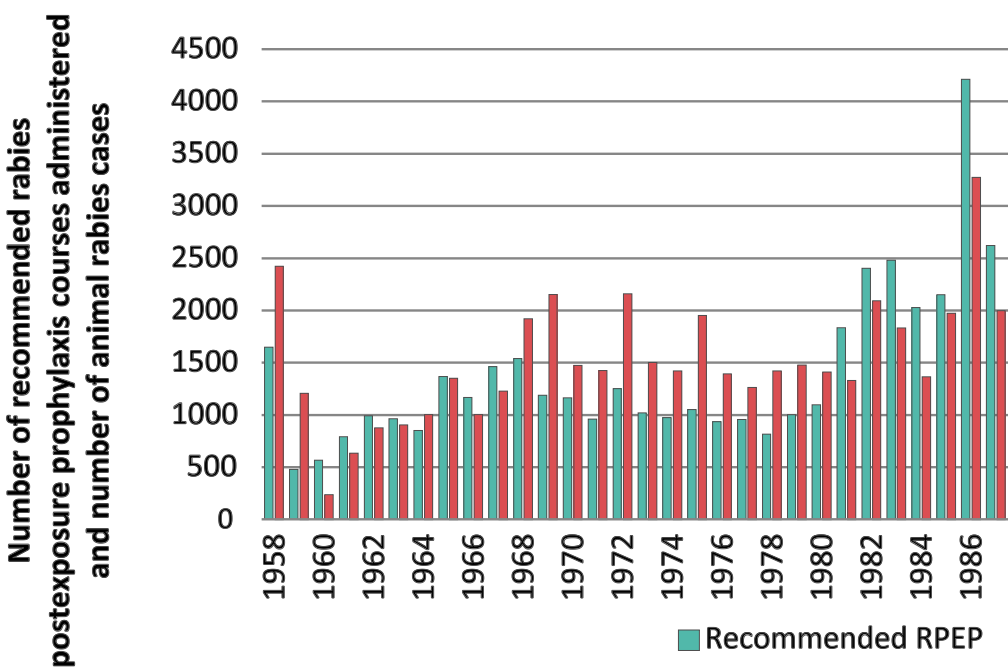

$\square$ Recommended RPEP

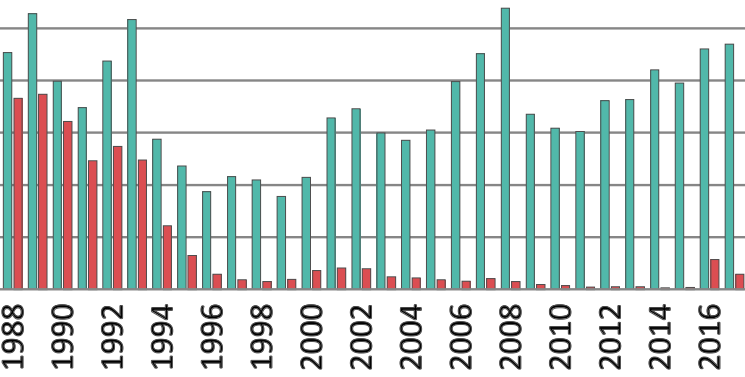

Animal Rabies Cases

Abbreviation: RPEP, rabies postexposure prophylaxis

a Rabid animal data for 1958-2013 obtained from published articles (4-6). For 2014-2017, animal rabies case data were obtained from the Canadian Food Inspection Agency (3)

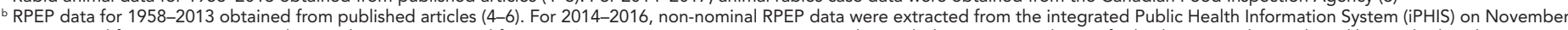

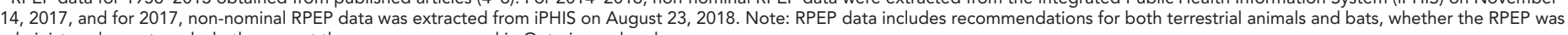
administered or not, and whether or not the exposure occurred in Ontario or elsewhere 


\section{Methods}

\section{Data sources}

Rabies postexposure prophylaxis data

In Ontario, incidents involving exposure to a potentially rabid animal for which RPEP is recommended (regardless of whether it is administered or not) are entered into the integrated Public Health Information System (iPHIS) by the PHU where the client resides (12). In iPHIS, PHUs are prompted to populate a number of fields with details relating to the incident. These include information about the individual exposed, the circumstances of the exposure, the animal (including whether it is available for observation and its vaccination status, if known), and if RPEP is recommended. An open-text comment section allows adding more details about the case.

iPHIS RPEP data for 2014 to 2016 were extracted on November 14, 2017. Only records with RPEP recommendations for terrestrial animal exposures that occurred in Ontario were included in the analyses. These included exposures to Ontario species that are known rabies reservoirs (i.e. raccoons, foxes and skunks) and bridge vectors (i.e. dogs, cats and livestock, including cattle, horses, sheep, goats and llamas). Excluded were records for exposures that occurred outside of Ontario; to animal species that were not terrestrial animals; or to animal species listed as "unknown" unless details in the comments section indicated that these were terrestrial animals and did not indicate the exposure occurred outside of Ontario.

\section{Population data}

Population estimates for each PHU from 2014 to 2016 were obtained from IntelliHEALTH Ontario $(13,14)$.

\section{Animal data}

The Ministry of Natural Resources and Forestry provided data on the year of the last confirmed terrestrial rabid animal reported for each PHU as of 2016 (personal communication, Stevenson B, Ministry of Natural Resources and Forestry, June 29, 2017).

\section{Analyses}

RPEP recommendation rates by PHU were calculated for the years 2014 to 2016. We used each PHU's annual RPEP data for exposures to terrestrial animals as the numerator and the population estimate for that PHU as the denominator, and graphed the results.

The number of years since the last rabid terrestrial animal was identified in each PHU relative to 2016 was also calculated. PHUs were categorized into one of the following five year intervals according to most recent report of a rabid terrestrial animal: 2016 or four preceding years, 6-10 years, 11-15 preceding years and more than 15 preceding years. We graphed 2016 RPEP recommendation rates for exposures to terrestrial animals by $\mathrm{PHU}$, classifying each $\mathrm{PHU}$ by their category with respect to the number of years since the last terrestrial rabid animal was identified in that PHU area relative to 2016. Microsoft Excel [version 2010; Microsoft Corporation, Redmond, Washington, United States (US)] was used to analyse the data and generate graphs. Mapping and spatial data preparation of these data were performed using ArcMap (version 10.3; ESRI, Redlands, California, US) geographic information systems (GIS) software.

\section{Results}

The annual RPEP recommendation rates for terrestrial animal exposures by PHU for 2014 to 2016 ranged from 3.0 to 35.2 per 100,000 persons, with a median of 11.9 RPEP recommendations per 100,000 persons. The annual rates of RPEP recommendations tended to be fairly consistent, with 10 PHUs having RPEP rates above the median and 15 having rates below the median, for all three years (Figure 3).

Figure 3: Annual rabies postexposure prophylaxis recommendation rates for terrestrial animal exposures by public health unit ${ }^{a}$, Ontario, 2014-2016

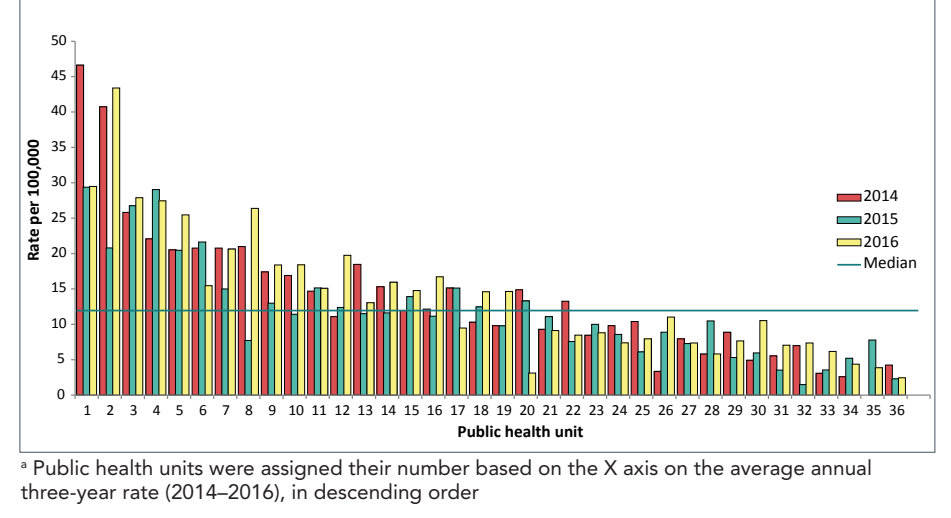

In 2016, ten PHUs had not identified a rabid terrestrial animal in their jurisdiction for more than 15 years (Figure 4). Five of these PHUs had RPEP recommendation rates above the provincial

Figure 4: Last confirmed terrestrial animal rabies case by public health unit, Ontario

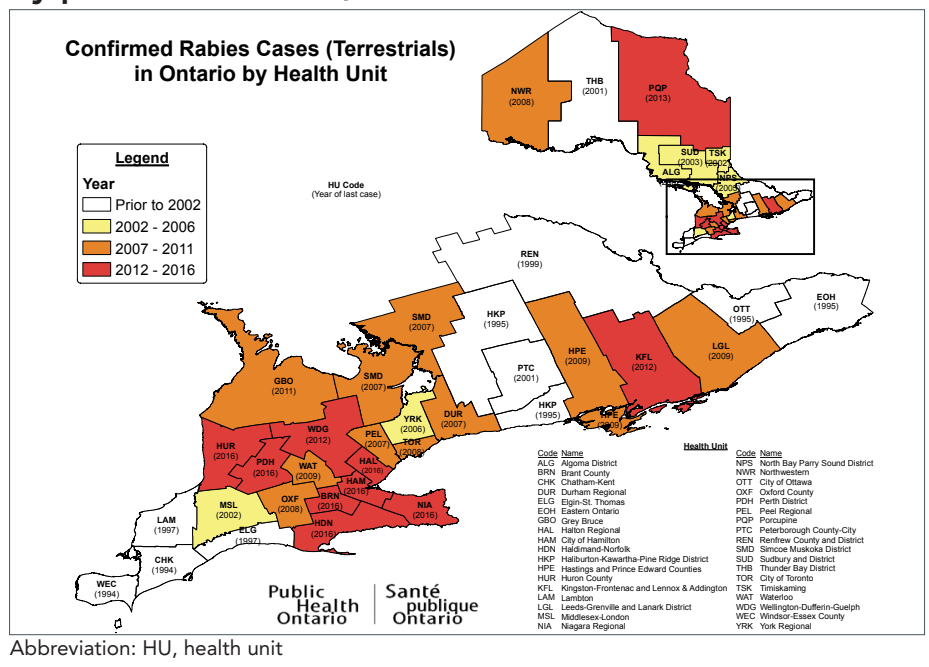


median. Two other PHUs, neither of which had identified a rabid terrestrial animal within the previous 11-15 years, also had RPEP rates above the median (Figure 5).

Figure 5: Rabies postexposure prophylaxis recommendation rates for terrestrial animal exposures within Ontario by public health unit ${ }^{\mathrm{a}}$ for 2016 and number of years since the last terrestrial rabies case relative to 2016

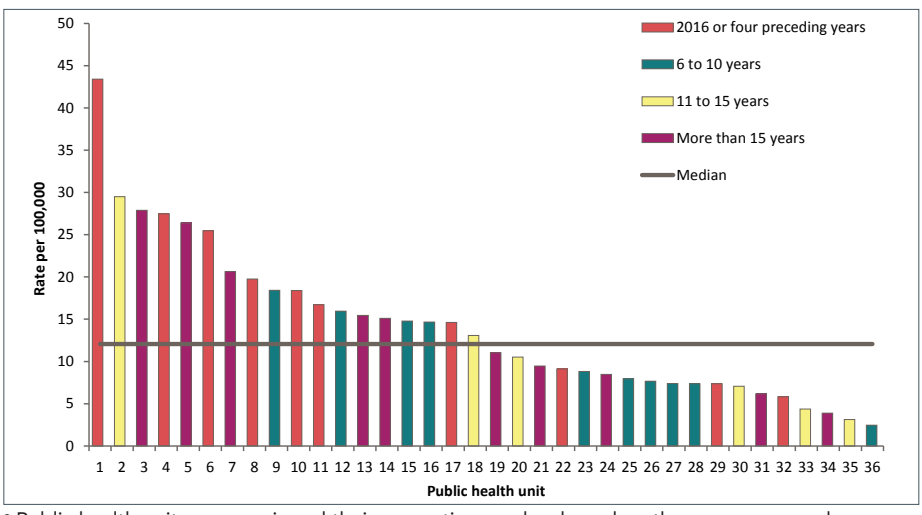

Public health units were assigned their respective number based on the average annual three-year rate (2014-2016) (shown in Figure 3), in descending order

\section{Discussion}

Since approximately the year 2000, the number of RPEP courses recommended in Ontario has been high compared to the number of rabid animals, even considering the epizootic in central west Ontario that was identified in late 2015 (Figure 2). Since 2013, risk assessments to determine the need for RPEP have been recommended if a person is exposed to dogs, cats or ferrets that are not available for subsequent observation. Implementation of a risk assessment can potentially optimize the number of courses of RPEP recommended by PHUs, particularly in those PHUs with no recent rabid terrestrial animals.

The 2014-2016 annual RPEP recommendation rates for exposures to terrestrial animals in Ontario varied markedly between PHUs, from 3.0 to 35.2 per 100,000 persons. However, in general, each PHU's annual RPEP rate remained relatively consistent over the three study years. A PHU's RPEP rate may be influenced by a number of factors, including the likelihood of human exposures to potentially rabid animals, the likelihood of these exposures being reported to the $\mathrm{PHU}$, and the risk assessment performed by the PHU in consultation with the health care provider. Data are not available to assess the likelihood of exposure to potentially rabid animals by $\mathrm{PHU}$, or the likelihood of reporting these exposures, or how the risk assessment was conducted. Nevertheless, data on confirmed cases of rabid animals within a PHU's jurisdiction, an important factor to consider when conducting a risk assessment, are available. Our analysis found that the recency of the last rabid terrestrial animal did not seem to be associated with the respective RPEP recommendation rates by PHU. Five PHUs had RPEP rates for terrestrial animals above the provincial median despite not reporting a rabid terrestrial animal in more than 15 years.

One possible explanation for the lack of association between RPEP recommendation rates for terrestrial animals and recency of terrestrial animal rabies could be that instead of conducting a risk assessment when an animal was not available for observation, some PHUs automatically recommend RPEP, consistent with the Canadian and Ontario recommendations prior to 2013. Another possible explanation for this lack of association is that the occurrence of terrestrial animal rabies is not heavily weighted when assessing the rabies risk after a terrestrial animal exposure when the animal is not available for observation.

The possibility that rabies may arise from sources outside the PHU's jurisdiction, because of incursions of rabid animals from adjacent areas, translocated rabid animals or adoption of rabid animals, may influence the decision to recommend RPEP, even though these events are infrequent. Incursions from adjacent areas as a possible source of introduction of terrestrial rabies only applies to PHUs that border another province or the US. An epizootic of raccoon rabies from incursion from adjacent areas in the US has not occurred in Ontario for more than 10 years and has never been demonstrated to have originated from neighbouring provinces (15). Four of the five PHUs that had not experienced a rabid animal in their jurisdiction more than 15 years and had RPEP recommendation rates above the median rate did not share a land border with the US.

Translocation of an imported rabid animal that results in an unrecognized epizootic is a concern. In recent years, there has been one recognized instance of translocation of a "hitch-hiking" raccoon that resulted in the current epizootic in central west Ontario (14). Adoption of a domestic animal with rabies has only been reported once in Ontario in recent years, when a puppy was transported from rural Ontario to a flea market in Toronto in 2008. The situation was quickly recognized and the large number of people exposed to the puppy were appropriately managed. In order to protect against rabies, dogs and cats more than three months old that are imported into Canada must be vaccinated (with minor exceptions) $(16,17)$, although on occasion, records have been falsified (18-21).

Another potential source of introduction of rabies into terrestrial animals comes from bats, which are known to carry rabies in Ontario. From 2000 to 2018, rabies strain typing in Ontario has identified 11 terrestrial animals with bat strain rabies: skunk (2001, 2004, 2016, 2018); cat (2002, 2004); fox (2003, 2009); raccoon (2002); bovine (2009); dog (2012) (personal communication, Gagnon R, Ministry of Natural Resources and Forestry, March 20, 2018). There is no evidence of transmission between animals based on the lack of identified clustering of any animals in time. In addition, a literature search revealed only one article that described the transmission of bat strain rabies among terrestrial animals, in skunks in Arizona (22). The transmission 
of bat strain rabies from terrestrial animals to humans also appears to be very rare: only three articles, all from South America, definitively identified the transmission of bat strain rabies to four humans via cats (23-25). These bats were vampire bats (Desmodus rotundus), which feed on mammalian blood, increasing the probability of infection in terrestrial animals; bats in Ontario feed on insects.

\section{Limitations}

There are a number of limitations in our analyses by PHU. As previously mentioned, we cannot assess other factors that may affect RPEP rates, such as the extent of human exposure to terrestrial animals and subsequent reporting to PHUs. PHUs frequently do not enter information on RPEP recommendations when it is not actually administered. Further, it is possible that the exposure occurred outside of the PHU's geographical boundary. With regard to rabid terrestrial animals, the data are limited by the extent of surveillance in the particular geographic area. In general, animals are tested for rabies when there has been a potential human exposure or during epizootics. It is therefore possible that rabid terrestrial animals in a PHU jurisdiction may be missed.

An additional limitation is that our analysis considered the timing of the last rabid terrestrial animal but did not consider the incidence rates at the time of that last rabid animal (i.e. it did not consider if there was one rabid animal versus multiple rabid animals) and we did not incorporate the recency of terrestrial animal rabies in adjacent PHUs into the analysis, both of which are factors that may impact the risk assessment.

\section{Conclusion}

Human rabies acquired from terrestrial animals has not occurred for more than 50 years in Canada. Canadian and Ontario guidelines recommend a risk assessment be conducted when a person is bitten or scratched by a dog, cat or ferret that is not available for a subsequent 10-day observation period. Consideration of the timing of the most recent rabid terrestrial animal in the geographic area is an important factor in determining the need for RPEP in the risk assessment.

RPEP administration rates for terrestrial animal exposures by PHU tend to be fairly consistent within each PHU when measured over a three-year period. Of the ten PHUs that had not had a rabid terrestrial animal in their area for more than 15 years, five had RPEP recommendation rates for terrestrial animal exposures above the provincial median RPEP recommendation rate. Along with other factors, consideration of the occurrence of rabies in terrestrial animals in a jurisdiction can assist in the risk assessment of dogs, cats or ferrets that are not available for observation.

\section{Authors' statement}

DM - Conceptualization, analysis and interpretation of data, drafting and revising the paper

LF - Analysis and interpretation of data, drafting and revising the paper

SJ - Analysis and interpretation of data, drafting the paper SB - Analysis and interpretation of data, revising the paper BW - Conceptualization, interpretation of data, drafting and revising the paper

\section{Conflict of interest}

None.

\section{Funding}

This work was supported by Public Health Ontario.

\section{Acknowledgements}

We wish to thank K Middel and B Stevenson from the Ministry of Natural Resources and Forestry for providing animal rabies data as well as interpretation of the data and findings, the Public Health Units in Ontario for entering rabies postexposure prophylaxis data in integrated Public Health Information System (iPHIS) and S Massarella (Public Health Ontario) for undertaking the literature search and C Martel (Public Health Ontario) for reviewing the translation.

\section{References}

1. Stevenson B. Raccoon strain rabies continues to climb. The rabies reporter. MNRF Publication 51709 2016;27(1):1-2. http://www.ontla.on.ca/library/repository/ ser/140213/2016//2016v27no01.pdf

2. National Advisory Committee on Immunization. Canadian Immunization Guide: Part 4 - Active vaccines: Rabies vaccine. Ottawa (ON): Public Health Agency of Canada; 2015. https:// www.canada.ca/en/public-health/services/publications/ healthy-living/canadian-immunization-guide-part4-active-vaccines.html

3. Canadian Food Inspection Agency. Rabies in Canada 2018. Ottawa (ON): Government of Canada; (Accessed 2019-0222). http://www.inspection.gc.ca/animals/terrestrial-animals/ diseases/reportable/rabies/2018-rabies-in-canada/ eng/1550860268670/1550860329289

4. Middleton D, Johnson KO, Rosatte RC, Hobbs JL, Moore SR, Rosella L, Crowcroft NS. Human rabies post-exposure prophylaxis and animal rabies in Ontario, Canada, 20012012. Zoonoses Public Health 2015 Aug;62(5):356-64. DOI PubMed 
5. Middleton D, Edwin JJ, Johnson KO, Warshawsky BF. A descriptive analysis of rabies post-exposure prophylaxis data: 2013, Ontario, Canada. Zoonoses Public Health 2018 Feb;65(1):e66-78. DOl PubMed

6. Nunan CP, Tinline RR, Honig JM, Ball DG, Hauschildt P, LeBer CA. Postexposure treatment and animal rabies, Ontario, 1958-2000. Emerg Infect Dis 2002 Feb;8(2):214-7. DOI PubMed

7. Ribadeau Dumas F, N'Diaye DS, Paireau J, Gautret P, Bourhy $\mathrm{H}$, Le Pen C, Yazdanpanah Y. Cost-effectiveness of rabies post-exposure prophylaxis in the context of very low rabies risk: A decision-tree model based on the experience of France. Vaccine 2015 May;33(20):2367-78. DOI PubMed

8. Population and Public Health Division, Ontario Ministry of Health and Long-Term Care. Rabies prevention and control protocol, 2013. Toronto (ON): Ontario Ministry of Health and Long-Term Care; 2013 Sep.

9. Population and Public Health Division, Ontario Ministry of Health and Long-Term Care. Rabies prevention and control protocol, 2018. Toronto (ON): Ontario Ministry of Health and Long-Term Care; 2018. http://www.health.gov.on.ca/en/ pro/programs/publichealth/oph_standards/docs/protocols_ guidelines/Rabies_Prevention_and_Control_Protocol_2018_ en.pdf

10. Infectious Disease Policy and Programs Unit, Public Health Policy and Programs Branch, Public Health Division, Ontario Ministry of Health and Long-Term Care. Guidance document for the management of suspected rabies exposures. Toronto (ON): Ontario Ministry of Health and Long-Term Care; 2013.

11. National Advisory Committee on Immunization. Canadian Immunization Guide. Part A - Active Vaccines: Rabies vaccine. Ottawa (ON): Public Health Agency of Canada; 2013.

12. Public Health Ontario. iPHIS user guide: Rabies post-exposure prophylaxis. Toronto (ON): Queen's Printer for Ontario; 2016.

13. IntelliHEALTH Ontario. Population estimates 1986-2015. Toronto (ON): Ontario Ministry of Health and Long-term Care (Accessed 2017-02-10).

14. IntelliHEALTH Ontario. Population estimates 2016-2017. Toronto (ON): Ontario Ministry of Health and Long-term Care (Accessed 2017-02-01).

15. Trewby H, Nadin-Davis SA, Real LA, Biek R. Processes underlying rabies virus incursions across US-Canada border as revealed by whole-genome phylogeography. Emerg Infect Dis 2017 Sep;23(9):1454-61. DOl PubMed

16. Canadian Food Inspection Agency. Importing or travelling with domestics dogs. Ottawa: Government of Canada. http://inspection.gc.ca/animals/ terrestrial-animals/imports/policies/live-animals/pets/dogs/ eng/1331876172009/1331876307796

17. Canadian Food Inspection Agency. Importing or travelling with domestic cats - three months of age or older from countries recognized by Canada as being rabies-free. Ottawa: Government of Canada. http:// www.inspection.gc.ca/animals/terrestrial-animals/ imports/policies/live-animals/pets/cats/rabies-free/ eng/1364954808466/1364954913271

18. Sinclair JR, Wallace RM, Gruszynski K, Freeman MB, Campbell C, Semple S, Innes K, Slavinski S, Palumbo G, Bair-Brake H, Orciari L, Condori RE, Langer A, Carroll DS, Murphy J. Rabies in a dog imported from Egypt with a falsified rabies vaccination certificate--Virginia, 2015. MMWR Morb Mortal Wkly Rep 2015 Dec;64(49):1359-62. DOI PubMed

19. Castrodale L, Walker V, Baldwin J, Hofmann J, Hanlon C. Rabies in a puppy imported from India to the USA, March 2007. Zoonoses Public Health 2008 Oct;55(8-10):427-30. PubMed

20. Centers for Disease Control and Prevention (CDC). Rabies in a dog imported from Iraq--New Jersey, June 2008. MMWR Morb Mortal Wkly Rep 2008 Oct;57(39):1076-8. PubMed

21. Hercules $Y$, Bryant NJ, Wallace RM, Nelson R, Palumbo G, Williams JN, Ocana JM, Shapiro S, Leavitt H, Slavinsk S, Newman A, Crum DA, Joseph BE, Orciari LA, Li Y, Yager P, Condori RE, Stauffer KE, Brown C. Rabies in a dog imported from Egypt - Connecticut, 2017. MMWR Morb Mortal Wkly Rep 2018 Dec;67(50):1388-91. DOI PubMed

22. Wenning K, Bergman DL, Worgess B, Levy C, Ferrat S, Marciniak J, Marciniak J, Rupprecht C, Slate D. Managing an outbreak of big brown bat variant rabies in terrestrial wildlife in Arizona. Conference: Rabies in the Americas 2010;XXI.

23. Paez A, Polo L, Heredia D, Nuñez C, Rodriguez M, Agudelo C, Parra E, Paredes A, Moreno T, Rey G. [An outbreak of human rabies transmitted by a cat in the town of Santander de Quilichao, Colombia, 2008]. Rev Salud Publica (Bogota) 2009 Dec;11(6):931-43. DOI PubMed

24. Bustos Claro MM, Álvarez AAA, Carrascal EJB, Martínez LGA, Benigni EM, Beltrán AFP, Ayala JAM. [Encephalitis due to rabies secondary to the bite of a cat infected with a rabies virus of Silvester origen] [in Spanish]. Infectio 2013;17(3):16770. DOI

25. Caicedo Y, Paez A, Kuzmin I, Niezgoda M, Orciari LA, Yager PA, Recuenco S, Franka R, Velasco-Villa A, Willoughby RE Jr. Virology, immunology and pathology of human rabies during treatment. Pediatr Infect Dis J 2015 May;34(5):520-8. DOI PubMed 